\title{
Gerechtigkeit im Wandel - Ein neuer Bewertungsmaßstab für soziale Ungleichheit \\ Christoph Butterwegge
}

Gerechtigkeit dient den Menschen als Kompass für die Entwicklung der Gesellschaft und als normativer Fixpunkt, um die soziale Ungleichheit zu beurteilen. Je nachdem, welcher Gerechtigkeitsbegriff in einer Gesellschaft vorherrscht, lässt sich die Kluft zwischen Arm und Reich politisch legitimieren oder skandalisieren. Mit den Plänen zum Um- bzw. Abbau des Sozialstaates häuften sich Bemühungen, die Gerechtigkeitsvorstellungen grundlegend zu verändern. ${ }^{1}$ Der allgemein gültige Gerechtigkeitsbegriff wurde dabei in dreifacher Hinsicht modifiziert: von der Bedarfs- zur „Leistungsgerechtigkeit“, von der Verteilungs- zur „Beteiligungsgerechtigkeit“ und von der sozialen zur „Generationengerechtigkeit“. Außerdem diskreditiert man soziale Gleichheit und Gerechtigkeit, indem die Freiheit geradezu mystifiziert und sehr viel stärker als bisher üblich im Sinne von "Privatinitiative“, „Eigenverantwortung“ bzw. „Selbstvorsorge“ (fehl)interpretiert wird.

\section{WOHLTATEN PRIMÄR FÜR WOHLHABENDE}

Peer Steinbrück, seinerzeit nordrhein-westfälischer Ministerpräsident, nahm eine totale Deformation des Gerechtigkeitsbegriffs vor und brach mit dem Sozialstaatspostulat des Grundgesetzes, als er die soziale Gerechtigkeit auf die Sorge des Staates um die Leistungsträger verkürzte: „Soziale Gerechtigkeit muss künftig heißen, eine Politik für jene zu machen, die etwas für die Zukunft unseres Landes tun: die lernen und sich qualifizieren, die arbeiten, die Kinder bekommen und erziehen, die etwas unternehmen und Arbeitsplätze schaffen, kurzum, die Leistung für sich und unsere Gesellschaft erbringen. Um die - und nur um sie - muss sich Politik kümmern." ${ }^{2}$
Mit der besseren steuerlichen Absetzbarkeit von Kinderbetreuungskosten und dem Elterngeld bietet die Familienpolitik zwei Beispiele für die Transformation von Bedarfs- in Leistungsgerechtigkeit. Sozial benachteiligte Familien, die aufgrund ihres fehlenden oder zu geringen Einkommens keine Steuern zahlen, kommen gar nicht erst in den Genuss, ihre Kinderbetreuungskosten steuerlich absetzen zu können eine Maßnahme, die bezeichnenderweise im Gesetz zur steuerlichen Förderung von Wachstum und Beschäftigung enthalten ist. Besserverdienende, die sich eine Tagesmutter oder Kinderfrau leisten und zwei Drittel der Aufwendungen hierfür (bis zu $4.000 €)$ absetzen können, profitieren überdurchschnittlich von dieser Regelung. Das seit dem 1. Januar 2007 gezahlte Elterngeld wird als Lohnersatzleistung in Höhe von 67 \% des vorherigen Nettoeinkommens gezahlt und erst bei $1.800 €$ pro Monat gedeckelt. Transferleistungsempfänger und -empfängerinnen, die Kinder erziehen, haben dagegen Nachteile: Bisher erhielten Sozialhilfebezieher, Arbeitslose und Studierende das Erziehungsgeld in Höhe von $300 €$ pro Monat für zwei Jahre (oder als „Budget" in Höhe von $450 €$ ein Jahr lang). Elterngeld gibt es nur noch für ein Jahr und der Sockelbetrag, der allen Nichterwerbstätigen bzw. -fähigen gezahlt wird, liegt gleichfalls bei $300 €$ (oder bei $150 €$, wenn er auf zwei Jahre verteilt wird). Erwerbstätige Paare erhalten unter bestimmten Voraussetzungen zwei (Partner-)Monate zusätzlich, wenn sie sich die Erziehungsarbeit teilen, und auch erwerbstätige Alleinerziehende können das Elterngeld 14 Monate lang in Anspruch nehmen. Mithin erhalten Gutbetuchte auf Kosten von schlechter Gestellten mehr (Eltern-) Geld. Diese Regelung soll hoch qualifizierte, gut verdienende Frauen motivieren, (häufiger) ein Kind zu bekommen und anschließend möglichst schnell wieder in den Beruf zurückzukehren. Bekämpft wird nicht die wachsende Armut von, vielmehr die Armut an Kindern.

\section{MEHR BILDUNG STATT UMVERTEILUNG?}

Obwohl das Volksvermögen so groß und die Kluft zwischen Arm und Reich so tief ist wie nie zuvor, gilt die Forderung nach Umverteilung als ideologisch verstaubt. Verteilungsgerechtigkeit, ein traditionelles Ziel sozialstaatlicher Politik, die nicht auf Armutsbekämpfung reduziert werden darf, wird durch Teilhabe- oder Beteiligungsgerechtigkeit ersetzt. In der „zivilen Bürgergesellschaft", wie sie Gerhard Schröder vorschwebte, steht der Gerechtigkeitsgedanke zwar im Mittelpunkt. Er bezieht sich aber nicht auf Verteilungsgerechtigkeit, die dem früheren Bundeskanzler als von der gesellschaftlichen Entwicklung überholt gilt:

\footnotetext{
1 Butterwegge, Ch. (2006): Krise und Zukunft des Sozialstaates, Wiesbaden

2 Steinbrück, P. (2003): Etwas mehr Dynamik bitte. Soziale Gerechtigkeit heißt heute: Der Staat muss mehr Geld in Bildung und Familien investieren. Für Gesundheit, Alter und Pflege hingegen werden die Bürger stärker selbst vorsorgen müssen, in: Die Zeit vom 13.11.2003.
}

Christoph Butterwegge, Prof. Dr., Leiter der Abteilung Politikwissenschaft am Seminar für Sozialwissenschaften der Universität zu Köln. Arbeitsschwerpunkte: Soziale Gerechtigkeit, Kinderarmut und Rechtsextremismus. e-mail: ewf-politikwissenschaft@uni-koeln.de 
„Gerade weil (...) die Herstellung und Bewahrung sozialer Gerechtigkeit in einem umfassenden Sinne oberstes Ziel sozialdemokratischer Politik ist und bleibt, können wir uns nicht mehr auf Verteilungsgerechtigkeit beschränken. Dies geht schon deshalb nicht, weil eine Ausweitung der Sozialhaushalte nicht zu erwarten und übrigens auch nicht erstrebenswert ist. Für die soziale Gerechtigkeit in der Wissens- und Informationsgesellschaft ist vor allem die Herstellung von Chancengerechtigkeit entscheidend." 3

Deutlicher wurde der frühere Wirtschafts- und Arbeitsminister Wolfgang Clement, als er im Rahmen der sozialdemokratischen Programmdiskussion für ein „neues Godesberg“ seiner Partei warb: „Heute geht es um den Abschied vom Wohlfahrtsstaat und die Hinwendung zum sozialen Bildungsstaat. (...) Der Sozialstaat in seiner bisherigen Ausprägung und Ausstattung trägt nicht mehr, und wir können ihn auch nicht mehr finanzieren. Wir müssen ihn deshalb nicht bloß umbauen, er braucht ein neues Fundament, eine neue Statik.“4 Unter einem „sozialen Bildungsstaat" versteht Clement, dass für alle Bürger prinzipiell gleiche Chancen zur beruflichen Qualifikation, zu einer hochqualifizierten Aus- und Weiterbildung geschaffen werden, damit sie aus eigener Kraft und Kompetenz auf die sich ständig verändernden Anforderungen der Arbeitswelt reagieren können. „Schulische Bildung und berufliche Qualifikation, Wissenschaft und Forschung sind die Motoren des ökonomischen und sozialen Fortschritts. Sie führen in die Wissensgesellschaft des 21. Jahrhunderts. Da müssen wir investieren, statt immer mehr in ein soziales Netz, das um so fadenscheiniger wird, je mehr wir ,draufsatteln."

Viel entscheidender als Umverteilung von Geld sei, dass Menschen einen gleichberechtigten Zugang zu den Bildungsinstitutionen und zum Arbeitsmarkt erhalten, heißt es immer häufiger. Wir leben allerdings in einer Zeit, in der das Geld nicht nur in fast sämtlichen Lebensbereichen wichtiger als früher, sondern auch ungleicher verteilt ist denn je. Daher stellt sich die Frage, warum die Bedeutung des Geldes für die Teilhabe der Bürger und Bürgerinnen am gesellschaftlichen Leben gesunken sein soll. Damit sie frei sind von Not, ihre Bedürfnisse befriedigen und ihre Pläne verwirklichen können, brauchen die Menschen nach wie vor Geld, das sie bei Er- werbslosigkeit, Krankheit und im Alter als soziale bzw. Entgeltersatzleistung vom Sozialstaat erhalten müssen. Mehr soziale Gleichheit bzw. Verteilungsgerechtigkeit bildet geradezu die Basis für die Teilhabechancen benachteiligter Gesellschaftsschichten. Dies gilt beispielsweise für die (Aus-)Bildung und den Arbeitsmarkt. Ohne ausreichende materielle Mittel steht zum Beispiel für Erwerbslose die Chance, an Weiterbildungskursen teilzunehmen, um ihre persönlichen Arbeitsmarktchancen zu verbessern, nur auf dem Papier. Natürlich ist es wichtig, dass Erwerbslose möglichst umgehend einen geeigneten Arbeitsplatz finden. Verteidiger des Sozialstaates, wie man ihn bisher kannte, haben denn auch nie dafür plädiert, Leistungsempfänger und -empfängerinnen nur $\mathrm{zu}$ alimentieren und sie nicht möglichst gut weiterzuqualifizieren - dies passiert jedoch seit Inkrafttreten der Hartz-Gesetze immer weniger.

So sinnvoll die Erweiterung des Gerechtigkeitsbegriffs in Richtung von "Teilhabe-“ oder "Beteiligungsgerechtigkeit" sein mag, so wenig darf sie vergessen machen, dass dieser durch soziale Ungleichheit der Boden entzogen wird. Unglaubwürdig wird, wer Bildungs- als Sozialpolitik interpretiert und gleichzeitig von der Schule über den Weiterbildungssektor bis zur Hochschule alle Institutionen dieses Bereichs privatisieren möchte. Denn das heißt letztlich, sie für Wohlhabende und die Kinder besser situierter Familien zu reservieren. In einem solchen Bildungssystem stoßen Kinder nur noch auf Interesse, wenn sie (bzw. ihre Eltern) als zahlungskräftige Kunden auftreten. Schon bevor Studiengebühren eingeführt wurden und ihnen bald vielleicht neuerlich Schulgeld folgt, gab es (Weiter-)Bildung nicht umsonst. Es wirkt zweifellos kontraproduktiv, wenn die Lernmittelfreiheit beschnitten wird und (Schul-)Bibliotheken aus Kostengründen schließen müssen.

Die zunehmende Kinderarmut wird häufig auf Bildungsarmut reduziert. Dies trägt zur Pädagogisierung und ideologischen Entsorgung des Armutsproblems bei. Bildungs- und Sozialpolitik dürfen nicht gegeneinander ausgespielt werden. Es ist falsch, die Erstere als „modern“ und die Letztere als „überholt“ zu betrachten, weil sie einander bei der notwendigen Inklusion von Kindern aus unterprivilegierten Elternhäusern ergänzen. Christof Prechtl und Daniel Dettling beklagen jedoch in einem von ihnen herausgegebenen Sammelband „Für eine neue Bildungsfinanzierung“, dass die Bundesrepublik sechsmal soviel Geld für Soziales wie für Bildung aufwendet; für sie ist Letztere der Schlüssel, um (Kinder-) Armut zu bekämpfen: „Da zwischen Bildungsstand und Erfolg am Arbeitsmarkt ein klarer Zusammenhang besteht, produziert das deutsche Bildungswesen heute die Sozialfälle von morgen. Politisch bedeutet dies: Die Vermeidung von Bildungs-, nicht Einkommensarmut, ist die zentrale Herausforderung. "6 Hier unterliegen die Verfasser allerdings einem Irrtum: Was zum individuellen Aufstieg taugen mag, versagt als gesellschaftliches Patentrezept. Wenn alle Kinder mehr Bildung bekommen, konkurrieren sie um die wenigen Ausbildungsbzw. Arbeitsplätze möglicherweise nur auf einem höheren Niveau, aber nicht mit besseren Chancen. Um die Massenerwerbslosigkeit und die zunehmende (Kinder-)Armut als gesellschaftliche Phänomene zu beseitigen, müssen Arbeit, Einkommen und Vermögen umverteilt werden. ${ }^{7}$

\section{"GENERATIONENGERECHTIGKEIT" ALS POLITISCHER SCHLACHTRUF}

24 Bundestagsabgeordnete unter 40 Jahren von Bündnis 90/Die Grünen, FDP und CDU/CSU traten im Juli 2003 mit einem Memorandum „Deutschland 2020“ an die Öffentlichkeit, das unter Mitwirkung der unter anderem von den Metallarbeitgebern finanzierten Initiative Neue Soziale Marktwirtschaft (INSM), der Altana AG und dem Think Tank „res publica“ entstanden war. Das Memorandum forderte mehr Generationengerechtigkeit und wandte sich dagegen, Reformen zu verschleppen. Nötig sei eine Neudefinition von Gerechtigkeit, die nicht mehr „sozialstaatliche Transferge-

\footnotetext{
3 Schröder, G. (2000): Die zivile Bürgergesellschaft. Anregungen zu einer Neubestimmung der Aufgaben von Staat und Gesellschaft, in: Die Neue Gesellschaft/Frankfurter Hefte 4, S. 203.

4 Clement, W. (2006): Ein neues „Godesberg " für die SPD, in: Welt am Sonntag vom 14.5.2006. 5 Clement, W. (2006): Ebd.

6 Prechtl, Ch./Dettling, D. (2005): „Wachstum durch Bildung - Chancen für die Zukunft nutzen! “, in: Dies. (Hrsg.): Für eine neue Bildungsfinanzierung. Perspektiven für Vorschule, Schule und Hochschule, Wiesbaden, S. 9.

7 Butterwegge, Ch./Klundt, M./Zeng, M. (2005): Kinderarmut in Ost- und Westdeutschland, Wiesbaden, S. $277 \mathrm{ff}$
} 
rechtigkeit“ sein dürfe, sondern als „Teilhabegerechtigkeit" für den Zugang zum Arbeitsmarkt und zu ökonomisch tragfähigen Formen sozialer Absicherung sorgen müsse: „Wer heute die soziale Gerechtigkeit nur an der Höhe staatlicher Transfers mißt, der beschränkt damit die Teilhabegerechtigkeit unserer Kinder und Enkel." 8 Generationengerechtigkeit bedeute, dass die von der aktiven Bevölkerung geschaffenen Ressourcen gerecht verteilt würden und dass die Politik für eine Realisierung dieser Potenziale sorge.

Wer glaubt, dass es bei einer Umverteilung der sozialen Besitzstände und Risiken nach dem Maßstab größerer Gerechtigkeit zwischen Älteren und Jüngeren sowohl eine klare Gewinner- als auch eine klare Verlierergeneration gibt, sieht sich getäuscht. CDU-Generalsekretär Ronald Pofalla erklärte die Generationengerechtigkeit zum „entscheidende(n) Kriterium“ für den Um- bzw. Abbau des Sozialstaates, das offenbar Jungen wie Alten materielle Opfer abverlangt: „Jede Generation muß ihren Beitrag leisten, damit wir soziale Sicherheit heute und morgen gewährleisten können."9 Deshalb sei es wichtig, dass die Regelaltersgrenze für den Rentenbezug erhöht, das Umlageverfahren in der Sozialen Pflegeversicherung durch Kapitaldeckung ergänzt und im Gesundheitswesen mehr Wettbewerb eingeführt werde.

Durch das Schlagwort „Generationengerechtigkeit" wird die soziale Spaltung unserer Gesellschaft biologisiert, auf ein Verhältnis zwischen Alterskohorten reduziert und relativiert. Letztlich handelt es sich bei der Generationengerechtigkeit um ein Konstrukt, das bestimmten Kräften ganz unabhängig von deren Altersgruppenzugehörigkeit dazu dient, im Rahmen sozialökonomischer und verteilungspolitischer Konflikte ihre eigene Position zu bestimmen und gegenüber anderen zu verbessern. Der Begriff der „Generationengerechtigkeit" verhüllt, dass sich die soziale Ungleichheit seit geraumer Zeit innerhalb jeder Generation verschärft und die zentrale soziale Trennlinie nicht zwischen Alt und Jung, sondern immer noch, ja mehr denn je zwischen Arm und Reich verläuft. Oft scheint es, als sei der Antagonismus zwischen Kapital und Arbeit durch einen neuen Grundwiderspruch - nämlich zwischen Jung und Alt - abgelöst und Klassenkampf durch einen „Krieg der Generationen“ ersetzt worden. Damit lenkt man von den eigentlichen Problemen, wie zum Beispiel der ungerechten Einkommens- und Vermögensverteilung, $a b$.

Das verkrampfte Bemühen um mehr Generationengerechtigkeit, der noch nie so viel Beachtung zuteil wurde wie heute, überdeckt die in sämtlichen Altersgruppen, der ganzen Gesellschaft und der Welt wachsende soziale Ungleichheit. Auch die Struktur der Armutspopulation dürfte sich infolge zahlreicher Kürzungen im Sozialbereich in Richtung der Senioren verschieben: Dazu zählen die Riester-Reform, die Senkung des Rentenniveaus durch den sogenannten Nachhaltigkeits- und den Nachholfaktor, die Erhöhung des Kranken- und des Pflegeversicherungsbeitrages vor allem für Betriebsrentner und -rentnerinnen, die nachgelagerte Rentenbesteuerung, die Verringerung des Schonvermögens von Langzeitarbeitslosen durch „Hartz IV“ und die Absenkung der Rentenversicherungsbeiträge, die für sie entrichtet werden. $\mathrm{Zu}$ sätzlich verstärkt wird das Problem durch die Zunahme diskontinuierlicher Erwerbsverläufe und von Scheidungen sowie durch die steigende Anzahl nicht eigenständig gesicherter Frauen.

Wenn ein Wohlfahrtsstaat demontiert wird, seine Transferleistungen für Bedürftige gesenkt und die gültigen Anspruchsvoraussetzungen verschärft werden, obwohl das Bruttoinlandsprodukt wächst und der gesellschaftliche Reichtum zunimmt, kann weder von sozialer noch von Generationengerechtigkeit die Rede sein. Denn offenbar findet eine Umverteilung statt, von der gerade die Mitglieder bedürftiger Alterskohorten nicht profitieren. Die am 9. März 2007 im Bundestag beschlossene Erhöhung des gesetzlichen Renteneintrittsalters von 65 auf 67 Jahre verschlechtert eher die Arbeitsmarktchancen künftiger Generationen statt Vorteile für diese mit sich zu bringen. Wer in den Ruf nach „Generationengerechtigkeit" einstimmt, müsste überhaupt darum bemüht sein, dass Heranwachsende auch später noch einen hoch entwickelten Wohlfahrtsstaat und das früher gewohnte Maß an sozialer Sicherheit vorfinden, statt Letztere immer mehr $\mathrm{zu}$ beschneiden und die Menschen der privaten Daseinsvorsorge zu überantworten.

Nicht nur die Renten, sondern auch die öffentlichen Haushalte sind ins Visier von „Experten“ geraten, die mehr Generationengerechtigkeit verlangen. In neoliberalen Kreisen wird gern behauptet, dass künftige Generationen hohe Schuldenberge ab- zutragen hätten, wozu sie weder willens noch in der Lage wären. Doch dieser Schuldendienst lastet nur auf einem Teil der kommenden Generationen; ein anderer erhält mehr Zinsen aus (geerbten) Schuldverschreibungen des Staates, als er selbst an Steuern zahlt, und profitiert dadurch sogar von heutigen Budgetdefiziten.

Es mutet beinahe kurios an, wenn Bundeskanzlerin Angela Merkel die Erhöhung der Mehrwert- und Versicherungssteuer von 16 auf $19 \%$, die seit dem 1 . Januar 2007 Normal- und Geringverdienerfamilien mit vielen Kindern besonders hart trifft, als „moralische Verpflichtung“ gegenüber künftigen Generationen legitimiert, denen dadurch angeblich der „Marsch in den Schuldenstaat" erspart bleiben soll. Ronald Pofalla, der die Debatte über ein neues Grundsatzprogramm seiner Partei organisiert, begründet dieses Gerechtigkeitsverständnis damit, dass man für jede Generation „eine faire Balance zwischen Eigenverantwortung und Solidarität " finden müsse: „Alte Gerechtigkeitspolitik ist auf die Gegenwart und auf horizontale Umverteilung beschränkt. Sie führt zu einer immer stärkeren Belastung der kommenden Generationen. Die neue Gerechtigkeit muß Lasten und Leistungen von heute und morgen zwischen Alt und Jung fair verteilen. Deshalb ist es richtig, daß die Union die Konsolidierung des Haushalts vorantreibt und damit die Schuldenlast unserer Kinder zurücknimmt, auch wenn dies heute mit harten Einschnitten für alle verbunden ist." 10

\section{„FREIHEIT “ UND "EIGENVERANTWORTLICHKEIT“}

Im Konzept eines „aktivierenden Sozialstaates", das ihn von den Aufgaben eines Bedürftige und Benachteiligte alimentierenden Fürsorgestaates entbinden möchte, spielen „Eigenverantwortung“, „Selbstvorsorge" und „Privatinitiative" eine Schlüsselrolle. Dabei geht es um eine „Neujustierung des Verhältnisses von Individuum und Staat", mithin um die Frage, ob Letzte-

\footnotetext{
8 „Deutschland 2020“. Für mehr Generationengerechtigkeit: Reformen nicht auf morgen oder übermorgen verschieben! Ein Memorandum der jungen Abgeordneten des Deutschen Bundestages, Berlin, 21. Juli 2003, S. 3

9 Pofalla, R. (2006): Neue Gerechtigkeit durch mehr Freiheit, in: FAZ vom 4.1.2006.

10 Pofalla, R. (2006): Ebd.
} 
rer die Menschen als mündige Bürger und Bürgerinnen, Bittsteller oder Kunden behandelt. Das bisherige Gemeinwesen droht in einen Wohlfahrtsmarkt sowie einen Wohltätigkeits-, Almosen- bzw. Suppenküchenstaat aufgespalten zu werden: Auf dem Wohlfahrtsmarkt kaufen sich die Bürger und Bürgerinnen, die es sich finanziell leisten können, soziale Sicherheit (z. B. Altersvorsorge durch private Versicherungen). Dagegen stellt der postmoderne Sozialstaat nur noch Minimalleistungen bereit, die euphemistisch "Grundsicherung" genannt werden. Sie bewahren die Menschen zwar vor dem Verhungern und Erfrieren, überlassen sie jedoch der Privatwohltätigkeit. Man spricht von „Eigenverantwortung", meint aber häufig nur eine Zusatzbelastung für Arbeitnehmer und Rentner, während die Arbeitgeber von Sozialversicherungsbeiträgen (Lohnnebenkosten) entlastet werden.

Gegenwärtig akzentuiert man nicht nur innerhalb der CDU, die ihre Programmdebatte unter das Motto „Neue Gerechtigkeit durch mehr Freiheit" gestellt hat, sondern auch innerhalb der Sozialdemokratie - dem neoliberalen Zeitgeist folgend - immer stärker die Freiheit. So kons- tatierte Gerhard Schröder in einem Essay zum 140. Jahrestag der Gründung seiner Partei: „Unser oberstes Leitbild ist die Freiheit der Menschen, ihr Recht auf ein Leben in Würde, Selbstbestimmung und freier Entfaltung ihrer Fähigkeiten in einem solidarischen Gemeinwesen." "In seiner als „Agenda 2010" bekannt gewordenen Regierungserklärung sprach Schröder am 14. März 2003 nicht weniger als $18 \mathrm{Mal}$ von „(Eigen-)Verantwortung“, in seiner Rede auf dem Berliner Sonderparteitag der SPD am 1. Juni 2003 sogar 19 Mal von „(Wahl-) Freiheit".

Die schrittweise Liquidation des Sozialstaates erhält ihre Legitimation, indem man sie als „Befreiung“ bevormundeter Bürger und Bürgerinnen feiert - dies kann nur perfide genannt werden. In einer wohlfahrtsstaatlichen Demokratie ist Freiheit die Möglichkeit der Schwächsten, über ihr Leben selbst zu bestimmen, statt unabhängig von der beruflichen Qualifikation wie der familiären Situation jeden Arbeitsplatz annehmen zu müssen. Sie meint aber gerade nicht die Möglichkeit von Begüterten und Spitzenverdienern, sich den allgemeinen Verpflichtungen zu entziehen. Wahlfreiheit kann deshalb nicht heißen, dass sich junge, gut verdienende und gesunde Arbeitnehmer durch die Option für preiswerte Spezialtarife ihrer Krankenkasse aus der sozialen Verantwortung stehlen. Vielmehr muss Wahlfreiheit darin bestehen, dass sich Alleinerziehende für Teilzeitarbeit entscheiden können, ohne dadurch noch Jahrzehnte später gravierende Nachteile bei der Bemessung ihrer Altersrente zu haben.

Die gezielte Aufwertung der Eigenverantwortung kaschiert letztlich nur, dass der Sozialstaat zunehmend aus seiner im Grundgesetz fixierten Verantwortung für das Wohl seiner Bürger entlassen wird. Schließlich ist die Rückverlagerung von Verantwortung auf die Familie und das Individuum, welches die Standardlebensrisiken wieder selbst trägt, in einer hoch entwickelten, arbeitsteilig organisierten und extrem ausdifferenzierten Gesellschaft anachronistisch, führt sie doch zu deren Entsolidarisierung, Pauperisierung größerer Teile der Bevölkerung und sozialer Polarisierung.

11 Schröder, G. (2003): Das Gestalten der Zukunft braucht den Mut zur Veränderung, in: Neue Gesellschaft/Frankfurter Hefte 5, S. 7.

\section{Betriebliche und überbetriebliche Interessenvertretung - Ergebnisse aus dem IAB-Betriebspanel 2005}

In Heft 2/2007 der WSI-Mitteilungen wurde bereits über aktuelle Ergebnisse aus dem IAB-Betriebspanel zur Tarifbindung der Betriebe und zur Nutzung von Öffnungsklauseln berichtet. ${ }^{1}$ Die dort präsentierten Zahlen werden nun ergänzt um Informationen zur zweiten tragenden Säule im bundesdeutschen dualen System der Interessenvertretung - der betrieblichen Mitbestimmung. Angesichts des zunehmenden öffentlichen und wissenschaftlichen Interesses an den Arbeitsbeziehungen in betriebsratslosen Betrieben sollen auch Daten zu betriebsspezifischen Formen der Mitarbeitervertretung vorgestellt und ein Blick auf das Zusammenspiel von betrieb- licher und überbetrieblicher Interessenvertretung geworfen werden.

\section{AKTUELLE ERGEBNISSE ZUR \\ BETRIEBLICHEN INTERESSENVERTRETUNG}

Einen Eindruck der quantitativen Reichweite der betrieblichen Mitbestimmung, das heißt der Verbreitung von Betriebsräten, vermittelt Tabelle 1 für die Privatwirtschaft West- und Ostdeutschlands (Betriebe ab fünf Beschäftigte). Hinsichtlich des Anteils der Betriebe mit gesetzlich legitimierter Interessenvertretung wird mit ca. $11 \%$ in beiden Landesteilen auch 2005 wie- der das Niveau der Vorjahre erreicht. Gleiches lässt sich für den Anteil der Beschäftigten in Betrieben mit Betriebsrat sagen

\footnotetext{
1 Kohaut, S. (2007): Tarifbindung und tarifliche Öffnungsklauseln: Ergebnisse aus dem IAB-Betriebspanel 2005, in: WSI-Mitteilungen 2, S. 94-96.
}

Peter Ellguth, wissenschaftlicher Mitarbeiter im Institut für Arbeitsmarkt- und Berufsforschung (IAB) der Bundesagentur für Arbeit in der Projektgruppe IAB-Betriebspanel. Arbeitsschwerpunkte: Arbeitsbeziehungen und betriebliche Arbeitszeitpolitik. e-mail: peter.ellguth@iab.de 
(47 \% in West- und $39 \%$ in Ostdeutschland). Die weitgehende Stabilität der quantitativen Basis der Mitbestimmung auf betrieblicher Ebene zeigt sich damit auch in den aktuell verfügbaren Zahlen.

Die hinlänglich bekannten größenspezifischen Unterschiede in der Verbreitung von Betriebsräten bilden sich ebenfalls wieder ab. Nur knapp jeder 14. Kleinbetrieb (bis 50 Beschäftigte) verfügt über einen Betriebsrat. Unter den Großbetrieben (über 500 Beschäftigte) ist die Existenz eines Betriebsrats dagegen die Regel und erreicht Anteilswerte von $89 \%$ in Westdeutschland und $86 \%$ in Ostdeutschland. Prägt in betrieblicher Perspektive die große Zahl der Kleinbetriebe das Gesamtbild, so relativiert sich deren Gewicht natürlich, wenn man die Anzahl der hier Beschäftigten betrachtet. Die geringere Verbreitung von Betriebsräten im mittel- und großbetrieblichen Segment Ostdeutschlands manifestiert sich in der dort rund 8 Prozentpunkte niedrigeren Reichweite der betrieblichen Mitbestimmung (Tabelle 1).

Ebenso bestätigen sich die erwarteten starken Unterschiede zwischen einzelnen Branchen, und das sowohl in Betriebs- als auch Beschäftigtenperspektive (Tabelle 2). Nach wie vor sind betriebliche Interessenvertretungen besonders stark im traditionell mitbestimmten Bereich Bergbau/ Energie- und Wasserversorgung und im Kredit- und Versicherungsgewerbe. Das untere Ende bilden wie gewohnt die vorwiegend kleinbetrieblich strukturierten Branchen des Dienstleitungsbereichs, des Handels und der Bauwirtschaft.

\section{BETRIEBSSPEZIFISCHE FORMEN DER MITARBEITERVERTRETUNG}

Alternativ zu oder auch neben einem Betriebsrat kann es in den Betrieben andere betriebsspezifische Formen der Mitarbeitervertretung geben. Solche Einrichtungen, seien es „Runde Tische“ oder „Belegschaftssprecher" etc., haben in Westdeutschland mit $11 \%$ eine wesentlich stärkere Verbreitung als in Ostdeutschland mit $5 \%$ (Tabelle 1). In auf Beschäftigte bezogenen Zahlen bedeutet dies, dass $13 \%$ der Beschäftigten in den alten und $8 \%$ in den neuen Bundesländern in entsprechenden Betrieben arbeiten. Diese Angaben beziehen sich ebenfalls auf die privatwirtschaftlichen Betriebe ab fünf Beschäftigte. Die Größenabgrenzung wurde gewählt, um eine Gegenüberstellung mit den Zahlen zur Verbrei-

Tabelle 1: Betriebsrat und andere Formen der Mitarbeitervertretung nach Betriebsgröße 2005

Basis: privatwirtschaftliche Betriebe mit mindestens 5 Beschäftigten*

\begin{tabular}{|c|c|c|c|c|c|c|}
\hline \multirow[b]{2}{*}{ Anteile in \% } & \multicolumn{5}{|c|}{ Betriebsgrößenklassen } & \multirow[b]{2}{*}{$\begin{array}{c}\text { Insgesamt } \\
\text { (ab 5 Besch.) }\end{array}$} \\
\hline & $\begin{array}{l}5 \text { bis } 50 \\
\text { Besch. }\end{array}$ & $\begin{array}{l}51 \text { bis } 100 \\
\text { Besch. }\end{array}$ & $\begin{array}{l}101 \text { bis } 199 \\
\text { Besch. }\end{array}$ & $\begin{array}{l}200 \text { bis } 500 \\
\text { Besch. }\end{array}$ & $\begin{array}{l}501 \text { u.m. } \\
\text { Besch. }\end{array}$ & \\
\hline \multicolumn{7}{|c|}{ Westdeutschland } \\
\hline Betriebe mit BR & 7 & 43 & 66 & 79 & 89 & 11 \\
\hline $\begin{array}{l}\text { Betriebe mit anderer } \\
\text { MA-Vertretung }\end{array}$ & 11 & 13 & 9 & 13 & 15 & 11 \\
\hline Beschäftigte mit BR & 12 & 45 & 67 & 81 & 92 & 47 \\
\hline $\begin{array}{l}\text { Beschäftigte mit anderer } \\
\text { MA-Vertretung }\end{array}$ & 13 & 13 & 9 & 13 & 16 & 13 \\
\hline \multicolumn{7}{|c|}{ Ostdeutschland } \\
\hline Betriebe mit BR & 7 & 44 & 61 & 79 & 86 & 11 \\
\hline $\begin{array}{l}\text { Betriebe mit anderer } \\
\text { MA-Vertretung }\end{array}$ & 5 & 8 & 12 & 9 & 15 & 5 \\
\hline Beschäftigte mit BR & 12 & 46 & 62 & 77 & 89 & 39 \\
\hline $\begin{array}{l}\text { Beschäftigte mit anderer } \\
\text { MA-Vertretung }\end{array}$ & 6 & 8 & 13 & 9 & 16 & 8 \\
\hline \multicolumn{6}{|c|}{$\begin{array}{l}\text { * ohne Landwirtschaft und Organisationen ohne Erwerbszweck. } \\
\text { Quelle: IAB-Betriebspanel 13. Welle West, 10. Welle Ost. }\end{array}$} & $\begin{array}{l}\text { Hans Böckler } \\
\text { Stiftung }\end{array}$ \\
\hline
\end{tabular}

tung eines Betriebsrats zu ermöglichen. Zudem spielen solche Einrichtungen in den Kleinstbetrieben ohnehin so gut wie keine Rolle. Das schon angesprochene $\mathrm{Ne}-$ beneinander von Betriebsrat und ,hausgemachter" Mitarbeitervertretung gibt es allerdings nur bei einem Anteil von weniger als $1 \%$ der Betriebe (ohne Tabelle). Wie nicht anders zu erwarten, kommt dies aber in größeren Betrieben deutlich häufiger vor (in Betrieben über 500 Beschäftigte bei ca. $8 \%)$.

Anders als bei den Betrieben, die einen Betriebsrat haben, gibt es bei Betrieben mit anderen Formen der Mitarbeitervertretung keine klare größenspezifische Verteilung. Eine gewisse Häufung scheint es allerdings im großbetrieblichen Segment zu geben. In der Branche, die andere Vertretungsformen am häufigsten ausweist, den sonstigen Dienstleistungen, arbeiten immerhin rund $20 \%$ der Beschäftigten in Betrieben mit einer solchen Einrichtung. In den übrigen Branchen bewegen sich die Anteilswerte um die 10 Prozentmarke (Tabelle 2). Diese Zahlen können natürlich keine Auskunft über die Ausgestaltung der betreffenden Gremien oder gar deren betriebspolitische Rolle geben. Dabei kann es sich um Einrichtungen handeln, deren Mitglieder gewählt oder aber von der Geschäftsleitung eingesetzt werden. Darüber hinaus gibt es sowohl reine Arbeitnehmerorgane als auch solche, in denen die Geschäftsleitung mit am Tisch sitzt. Ebenso können auf dieser 2 Basis keine Aussagen zum Verhältnis von gesetzlich legitimierter betrieblicher Interessenvertretung und betriebsspezifischen
Mitarbeitervertretungen getroffen werden. Dies muss weiteren Analysen vorbehalten bleiben. $^{2}$

\section{DAS ZUSAMMENSPIEL VON BETRIEBLICHER UND ÜBERBETRIEB- LICHER INTERESSENVERTRETUNG}

Abschließend soll noch ein gemeinsamer Blick auf die betriebliche und sektorale Ebene der (gesetzlich legitimierten) Interessenvertretung geworfen werden. Sowohl die seit Längerem anhaltende Tendenz einer Verbetrieblichung der Tarifpolitik als auch die immer wieder in die politischen Debatte eingeworfenen Vorschläge zur Erweiterung betrieblicher Handlungsspielräume setzen (mehr oder weniger stillschweigend) die Existenz eines kompetenten betrieblichen Verhandlungspartners voraus, der die Interessen der Arbeitnehmer bündelt und vertritt.

Damit gilt im Folgenden das Augenmerk vor allem den sogenannten betrieblichen Vertretungslücken, also einerseits Betrieben, die zwar tarifgebunden sind, aber über keinen Betriebsrat verfügen. Und andererseits geht es um sogenannte weiße Flecken in der Tarif- und Mitbestimmungslandschaft, wo es keinerlei gesetzlich

Vgl. hierzu auch Hauser-Ditz, A./Hertwig, M. Pries, L. (2006): Betriebsräte und andere Vertretungsorgane im Vergleich - Strukturen, Arbeitsweisen und Beteiligungsmöglichkeiten, in: WSIMitteilungen 9, S. 500-506. 
Tabelle 2: Betriebsrat und andere Formen der Mitarbeitervertretung nach Branchen 2005 Basis: privatwirtschaftliche Betriebe mit mindestens 5 Beschäftigten*

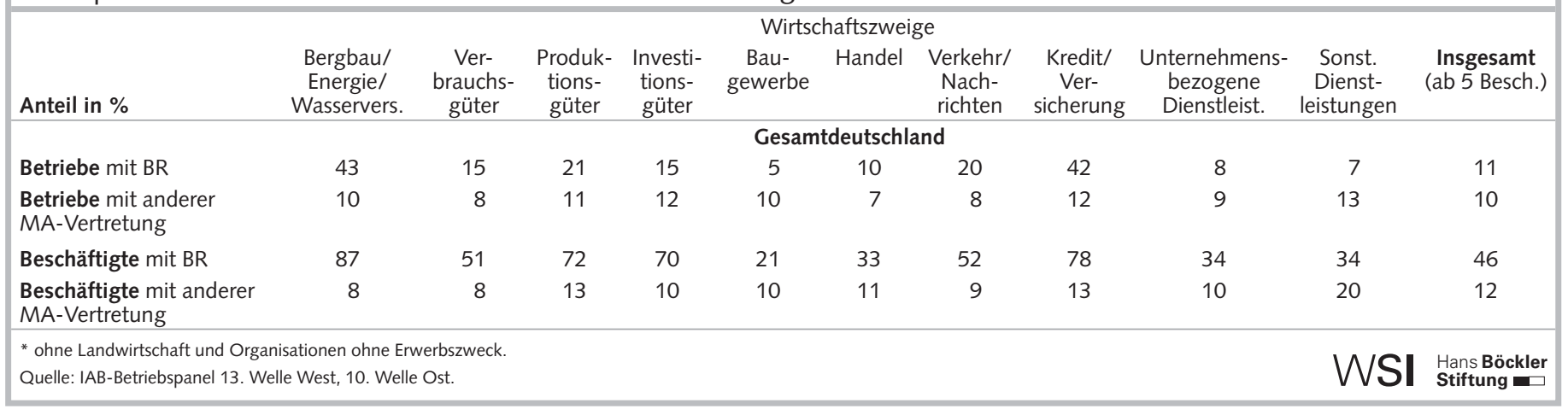

\begin{tabular}{|c|c|c|c|c|c|c|c|c|c|c|c|}
\hline & \multicolumn{6}{|c|}{ Westdeutschland } & \multicolumn{5}{|c|}{ Ostdeutschland } \\
\hline & 1996 & 1998 & 2000 & 2002 & 2004 & 2005 & 19961998 & 2000 & 2002 & 2004 & 2005 \\
\hline & \multicolumn{11}{|c|}{ Anteil der jeweils betroffenen Beschäftigten in \% } \\
\hline BR und Branchentarif & 41 & 39 & 37 & 37 & 35 & 33 & $28 \quad 24$ & 23 & 21 & 20 & 21 \\
\hline BR und Haustarif & \multirow{2}{*}{\multicolumn{2}{|c|}{$9 * * \begin{array}{l}6 \\
4\end{array}$}} & 6 & 6 & 6 & 7 & \multirow{2}{*}{$13 * *$} & 8 & 11 & 10 & 9 \\
\hline BR und kein Tarif & & & 6 & 6 & 6 & 6 & & 8 & 9 & 9 & 9 \\
\hline $\begin{array}{l}\text { Branchentarif und } \\
\text { kein BR }\end{array}$ & 27 & 27 & 24 & 24 & 24 & 23 & 22 & 17 & 14 & 16 & 16 \\
\hline Haustarif und kein BR & \multirow{2}{*}{\multicolumn{2}{|c|}{$23 * * \begin{array}{r}2 \\
21\end{array}$}} & 1 & 1 & 1 & 1 & \multirow{2}{*}{$36 * *$} & 4 & 4 & 4 & 4 \\
\hline kein Tarif und kein BR & & & 25 & 25 & 27 & 29 & & 40 & 42 & 42 & 41 \\
\hline Gesamt & 100 & 100 & 100 & 100 & 100 & 100 & 100 & 100 & 100 & 100 & 100 \\
\hline \multicolumn{12}{|c|}{$\begin{array}{l}\text { * ohne Landwirtschaft und Betriebe ohne Erwerbszweck. } \\
\text { ** Da sich die Abfrage der Geltung eines Haus- bzw. Firmentarifvertrags zwischen } 1996 \text { und } 1998 \text { geändert hat, können die } \\
\text { entsprechenden Werte erst von } 1998 \text { an ausgewiesen werden. Für } 1996 \text { werden deshalb die zusammengefassten Werte der } \\
\text { Betriebe ohne Branchentarif angegeben. } \\
\text { Quelle: IAB-Betriebspanel 1996-2005. }\end{array}$} \\
\hline
\end{tabular}

legitimierte Interessenvertretung gibt. ${ }^{3}$ Die präsentierten Zahlen beziehen sich ebenfalls auf die Betriebe der Privatwirtschaft ab fünf Beschäftigte. Eine Berücksichtigung der Kleinstbetriebe, die ja nicht zur Wahl eines Betriebsrats berechtigt sind, würde bei der gemeinsamen Betrachtung des Deckungsgrades von betrieblicher Mitbestimmung und Tarifbindung ein systematisch verzerrtes Bild liefern.

Wie sich auch in den aktuellen Zahlen bestätigt, ist nur eine Minderheit der in der Privatwirtschaft (ab fünf Beschäftigte) beschäftigten Arbeitnehmer in Betrieben tätig, die zur Kernzone des dualen Systems der Interessenvertretung zu zählen sind. Diese Zone umfasst ein Drittel der Beschäftigten in Westdeutschland und nur ein gutes Fünftel in Ostdeutschland (Tabelle 3). Bemerkenswert ist die mit $9 \%$ relativ große Bedeutung von Betrieben mit Haustarifen und einem Betriebsrat in den neuen Bundesländern. Von einer betrieblichen Vertretungslücke (Betrieb ist an einen Branchen- tarif gebunden, verfügt aber über keinen Betriebsrat) ist jeder vierte Beschäftigte in Westdeutschland, aber nur jeder sechste in Ostdeutschland betroffen. Diese vermeintlich günstigere Situation in den neuen Bundesländern ist allerdings nur der dort geringeren Reichweite der Tarifbindung geschuldet, die sich in deutlicher ausgeprägten „weißen Flecken der Tarif- und Mitbestimmungslandschaft “äußert. Während in Westdeutschland „nur“ 29 \% der Beschäftigten ohne Branchentarif und ohne Betriebsrat auskommen müssen, sind dies in Ostdeutschland $41 \%$.

Betrachtet man diese Zahlen über die Jahre hinweg, so zeigen sich einige bemerkenswerte Entwicklungen. Die Kernzone des dualen Systems ist seit 1996 in beiden Landesteilen deutlich geschrumpft. Während sich dieser Rückgang in Westdeutschland bis zum aktuellen Rand fortsetzt, bleiben die Anteile im Osten seit 2002 stabil, wenn auch auf deutlich niedrigerem $\mathrm{Ni}$ veau als im Westen.
Ebenfalls abgenommen hat in den letzten zehn Jahren der Umfang der betrieblichen Vertretungslücken. Dies ist aber wiederum keine Erfolgsmeldung, sondern muss im Zusammenhang mit den deutlich angewachsenen ,weißen Flecken in der Tarif- und Mitbestimmungslandschaft" gesehen werden. Ein weiteres Segment, das vor allem in Ostdeutschland Zuwächse zu verzeichnen hat, umfasst Betriebe, die nicht (mehr) an einen Branchentarif gebunden sind, aber über einen Betriebsrat verfügen. All diese Trends sind zum weitaus größten Teil dem Rückgang der Branchentarifbindung geschuldet bei parallel weitgehend stabiler Reichweite der betrieblichen Mitbestimmung. Was dabei durchgängig zu beobachten ist, ist die schon für die Kernzone des dualen Systems erwähnte Konsolidierung in Ostdeutschland seit 2002. Die weitere Entwicklung muss zeigen, ob es sich dabei vielleicht nur um eine Verschnaufpause im Erosionsprozess der überbetrieblichen Interessenvertretung handelt.

\footnotetext{
3 In der Diskussion um die Interessenvertretung in Betrieben ohne Betriebsrat wird immer wieder darauf hingewiesen, dass "betriebsratslos" nicht gleichbedeutend sei mit partizipationsfrei. Dem ist natürlich auch in Anbetracht der oben präsentierten Zahlen zu den betriebsspezifischen Formen der Mitarbeiterbeteiligung zuzustimmen. Allerdings können in dem hier erörterten Zusammenhang solche Einrichtungen angesichts ihrer fehlenden rechtlichen Ausstattung $\mathrm{m}$. E. nicht als funktionales Äquivalent zu einem Betriebsrat gelten. Für die getroffene Zuordnung der Betriebe (z. B. betriebliche Vertretungslücke) ist aus diesem Grund auf betrieblicher Ebene nur die Existenz bzw. das Fehlen eines Betriebsrats relevant.
} 